\title{
OTIOTOMICS
}

Revista de economía, empresa y sociedad

\section{¿Qué papel juega la marca de distribuidor en los surtidos de las cadenas de distribución alimentaria?}

\author{
Juan Garlos Gázquez-Abad \\ Profesor titular de la Universidad de Almería \\ Profesor colaborador de los Estudios de Economía y Empresa (UOC)
}

\begin{abstract}
RESUMEN Las marcas de distribuidor (MdD) se han convertido en un «fenómeno social y económico", especialmente en el contexto de la distribución con base alimentaria. Si bien es cierto que la crisis económica ha sido uno de los elementos que ha permitido el éxito de este tipo de marca, los minoristas han sido capaces de desarrollar enseñas con un elevado valor para el consumidor, lo que ha permitido que se hayan posicionado al mismo nivel que muchas de las marcas de fabricante tradicionales. Esto ha causado que muchos minoristas hayan optado por darle mayor protagonismo a su marca propia en detrimento de las marcas nacionales, de modo que la MdD se ha convertido en su principal «arma competitiva» frente a los fabricantes de las marcas nacionales más tradicionales. En este artículo se analiza el papel que la MdD juega en los surtidos de las cadenas de distribución alimentaria y hasta qué punto la apuesta por la marca propia que muchas cadenas han realizado puede ser perjudicial para los intereses del minorista.
\end{abstract}

PALABRAS CLAVE gestión minorista; surtido; marca de distribuidor; marcas nacionales

\section{The role of Private Labels in retailers' assortments: an analysis in the FMCG sector}

\begin{abstract}
Private labels (PLs) have become a «social and economic phenomenon», especially in the context of the fast moving consumer goods (FMCG) sector. Although the global economic crisis has boosted PLs' success, retailers have developed their own high-value brands. As a result, PL positioning is very close to that of (traditional) national brands competing in the sector. Because of such positioning, many retailers have emphasized their own brands on their shelves. PLs have become the most important competitive tool retailers employ against national brands. This paper analyses the role of PLs in retailers' assortments and whether many of retailers' assortment decisions might be detrimental to their own interests.
\end{abstract}

KEYWORDS retailing; assortment; private label; national brands 


\section{Introducción}

El sector de la distribución comercial ha sufrido una transformación importante en los últimos años, tanto en los procesos de comercialización como en el comportamiento de compra de los consumidores. Uno de los cambios más relevantes ha sido, sin lugar a dudas, la consolidación de las marcas de distribuidor (MdD) -especialmente en el contexto de la distribución con base alimentaria- que han alcanzado unas cifras de cuota de mercado muy elevadas a costa de las marcas de fabricante, lo que ha alterado profundamente la composición de los surtidos en los lineales de venta y la relación entre el minorista y los fabricantes.

En este trabajo se analiza, fundamentalmente en el contexto de la distribución con base alimentaria en España, la situación actual de las MdD y el papel que la mismas juegan no solo dentro de la oferta de referencias del minorista, sino, además, a nivel estratégico como herramienta competitiva y de negociación frente a otros minoristas y fabricantes.

\section{1. ¿Qué es la MdD? Evolución y situación actual}

Los productos con MdD son los que han sido fabricados por un determinado fabricante y son ofrecidas al consumidor bajo el nombre o marca de la empresa distribuidora, y no con el de la compañía que realmente los ha elaborado. La literatura recoge varias definiciones de MdD. Por ejemplo, Serra y Puelles (1993) se refieren a ellas como aquellas que, desvinculadas del fabricante que las produce, se comercializan por un distribuidor concreto que les da su nombre u otro distinto, y se encarga de realizar las labores de marketing. Casares y Rebollo (1996) afirman que las $\mathrm{MdD}$ se refieren a las de aquellos productos fabricados por un determinado industrial, que son ofrecidas al consumidor bajo el nombre o marca del distribuidor o detallista, que es quien realiza todas las labores de marketing. Para Puelles y Puelles (2003), las MdD son marcas cuya propiedad legal corresponde al distribuidor y de cuya fabricación, normalmente, se encarga un fabricante establecido. El fabricante sigue en su producción las especificaciones requeridas por el distribuidor en cuanto a la composición, calidad, diseño, envase, etc.

La American Marketing Association (AMA) se refiere a la MdD como aquella marca cuya propiedad y control corresponde al distribuidor, en contraposición al fabricante. El término no solo se aplica a la marca propiamente dicha, sino que se extiende al producto y su contenido. Frecuentemente es conocida como marca privada o etiqueta privada, aunque en castellano ${ }^{1}$ existen muchos otros vocablos que se han utilizado con mayor o menor acierto para referirse a tales marcas (por ejemplo, marcas propias de detallista, marcas blancas ${ }^{2}$, marca de enseña, marca de cadena propia, marca exclusiva, marca cedida, marca genérica, producto bandera, contramarcas).

Los supermercados fueron el formato base para el desarrollo de las MdD (Puelles et al., 2011). Los primeros indicios de los productos propiedad del distribuidor los encontramos en el Reino Unido en 1869, cuando la cadena Sainsbury lanzó su propia marca. En Francia, las primeras marcas de este tipo aparecieron en 1923, cuando la cadena Coop decidió registrar su marca para toda una variedad de productos. No obstante, la primera iniciativa importante de MdD tuvo lugar en Francia en 1976, con la introducción en los lineales de Carrefour de unos cincuenta productos sin marca, solamente identificados con su logotipo (Gázquez-Abad y Sánchez-Pérez, 2007).

Sin embargo, nada tienen hoy que ver las actuales marcas de distribuidor con aquellas «marcas blancas» de la década de los años sesenta y setenta, denominadas «de primera generación» y caracterizadas por envases muy sencillos y de color blanco, con etiquetas muy simples, que llegaron a España con los productos de la cadena de

1. En inglés sucede algo similar; los términos más empleados son: private labels, private brands, store brands, own labels, own brands, retailer's brands o home brands.

2. Este es, probablemente, el término más utilizado por los medios de comunicación en nuestro país para referirse a la MdD. El término marca blanca se debe a que las primeras marcas de distribuidor lanzadas al mercado utilizaban envases blancos con letras negras que indicaban la categoría del producto en cuestión. 
supermercados Simago ${ }^{3}$. Sin embargo, no fue hasta a partir de los años noventa, de la mano de las grandes superficies comerciales, cuando las MdD dieron un salto en calidad e imagen, comercializándose bien con el nombre del minorista (marca de distribuidor, propiamente dicha), o con nombres distintos (marca privada), gestionados por el distribuidor, quien se encargaba de todas las labores de comercialización y marketing (Puelles, 2011).

Europa lidera el sector de la MdD a nivel mundial, que es más fuerte en aquellas economías europeas más potentes. Las principales economías europeas (Suiza, Alemania, Francia, España, Reino Unido y Países Bajos), así como países como Portugal, Austria o Bélgica, cuentan con cuotas de mercado de la MdD por encima del 25\%. Según datos de IRI (2014), la cuota de mercado (valor) de la MdD en Europa es del 38,7\% (48,9\% en unidades). Por países, Suiza, junto con el Reino Unido y España, son los que ostentan los mayores valores de cuota de mercado de la MdD, con valores superiores (en el caso de Suiza y el Reino Unido) al 50\% (valor). No obstante, cabe señalar que en 2014 la cuota de mercado de la MdD en Europa descendió (0,1\%) por primera vez desde 2007, lo que en opinión de muchos expertos puede poner de manifiesto cierta saturación de los mercados, el logro de un «máximo» en la cuota de mercado de este tipo de marcas, y la recuperación del nivel de gasto y empleo a nivel mundial.

En España, según datos de IRI (2016), la cuota de mercado de la MdD (enero de 2016) es del 40,2\% (valor), y también aquí cabe señalar que en relación a enero de 2015, dicha cuota se ha visto reducida en un 1\%. Igualmente, las ventas de la MdD se han visto reducidas en un 1,7\% (valor) en ese mismo período. Dicha reducción ha sido aprovechada por las marcas de fabricante (o marcas nacionales), que han incrementado sus ventas en un 2,2\% (han sido las marcas que han aprovechado, en mayor medida, la lenta recuperación de la economía española). Del conjunto de categorías, cabe destacar que son los alimentos frescos y refrigerados los que mayor cuota de mercado de la MdD presentan (por encima, en la mayor parte de los casos, del $40 \%$ ). Por el contrario, las categorías de higiene y cuidado personal y las bebidas alcohólicas son las que presentan menores cifras de cuota de mercado de la MdD (por debajo, en la mayor parte de los casos, del 15\%).

\section{Importancia de la MdD en el surtido del minorista}

El surtido se configura, junto con la localización y el nivel de precios, como un criterio fundamental a la hora de explicar por qué el consumidor selecciona un establecimiento y no otro.

Aunque inicialmente los minoristas introdujeron su marca propia en los lineales como forma de completar su oferta llegando a los segmentos de precio más bajo, desde hace unos años la presencia de la MdD se ha convertido en la principal herramienta competitiva en manos de los distribuidores alimentarios frente al mayor poder tradicional que las marcas de fabricante o marcas nacionales han acumulado. Esta capacidad competitiva se expresa a partir de diversos aspectos:

- La MdD permite al minorista ejercer un mayor control sobre el espacio disponible en sus lineales (Amrouche y Zaccour, 2007). Esto es un elemento fundamental en la negociación con los fabricantes de marcas nacionales, ya que la posición de los productos en los lineales condiciona, y mucho, la probabilidad de que sean elegidos por parte del consumidor. En la medida en la que la relación con el fabricante es más beneficiosa para el minorista, los productos de aquel suelen ocupar mejores zonas/pasillos y suelen situarse en los niveles centrales (nivel de los ojos) de las estanterías, que son, normalmente, los más beneficiosos desde el punto de vista de la visibilidad y la probabilidad de elección ${ }^{4}$.

3. Simago se especializó durante los años sesenta, setenta y ochenta en ofrecer a los consumidores productos de bajo coste. Sus locales solían tener poco más de 1.000 metros cuadrados, generalmente repartidos entre bazar y supermercado. En 1997, Simago fue adquirida por la cadena de hipermercados Continente y sus establecimientos pasaron a operar bajo la marca Champion.

4. Si bien es cierto que el nivel central de los ojos suele ser el más beneficioso, esto también depende, en muchas ocasiones, de la categoría del producto en concreto. Por ejemplo, la mejor posición en el caso de los yogures suele ser algo más baja que el nivel de los ojos, dado que el consumidor presta mayor atención a la fecha de caducidad. 
- La MdD permite reforzar la imagen del establecimiento (Ailawadi y Keller, 2004). Muchos consumidores entienden la MdD como una extensión de la marca y la imagen del propio establecimiento. De hecho, ambos conceptos -MdD e imagen de establecimiento- se complementan mutuamente. Cuando los consumidores tienen una percepción positiva de la imagen del establecimiento, esto genera un efecto positivo en las marcas que comercializa dicha tienda (Dhar y Hoch, 1997). Aún más, cuando los consumidores no están familiarizados con la marca, la imagen del establecimiento es uno de los elementos fundamentales para hacer un juicio rápido sobre la MdD, por lo que puede afirmarse que la imagen del establecimiento tiene una relación directa y positiva con la intención del consumidor a la hora de comprar MdD. Cuanto más positiva sea la imagen del establecimiento, mayor será la intención de compra de la MdD (Grewal et al., 1998).

- La MdD permite incrementar el poder de negociación del minorista frente a los fabricantes (Baltas y Argouslidis, 2007). Es decir, en la medida en la que el minorista posea una marca propia poderosa y con un nivel de cuota de mercado importante, el nivel de «dependencia» del surtido respecto de las marcas nacionales será menor. Por el contrario, si la marca propia es poco preferida y valorada por parte del consumidor, el minorista se verá obligado a contar con un mayor número de marcas nacionales en su surtido para «atraer» a los consumidores al punto de venta, ya que con su propia marca se ve incapaz de hacerlo.

Dadas todas las «virtudes» competitivas que la marca propia ofrece al distribuidor, no es de extrañar que muchos grupos minoristas con base alimentaria hayan apostado claramente por sus marcas. Esta mayor apuesta por la MdD se ha visto incentivada por la recesión a la que se han visto sometidas muchas de las economías occidentales en los últimos años. En este sentido, la situación económica negativa reciente ha ofrecido a los minoristas la oportunidad de consolidar una mayor presencia de sus marcas propias. Así, las marcas propiedad del distribuidor, gracias a una mayor presencia en los lineales de venta de los minoristas y a una fuerte inversión en comunicación, han sabido transformar su posicionamiento, situándose como una alternativa óptima en la relación preciocalidad. Estas marcas propias se han ganado la confianza de un gran número de consumidores y, de hecho, se presentan en la actualidad como serias competidoras de las marcas de fabricante (Lamey et al., 2012), no ya solamente en relación al precio, sino incluso en otros aspectos relacionados con los beneficios que obtienen los consumidores; según Roger (2010), la propuesta de valor de las marcas de distribuidor es superior a las marcas de fabricante. Por todo ello, es previsible que las MdD se posicionen en las mentes de los consumidores como alternativas válidas incluso una vez que se supere la crisis económica. Según un informe de IRI (2013), el 60\% de los consumidores españoles de MdD comenzaron a consumirlas como consecuencia de la recesión económica, y solamente el $21 \%$ de ellos muestra intención de volver a comprar las marcas de fabricante (de precio superior) que compraban anteriormente una vez que la situación económica haya mejorado.

No obstante, y a pesar de ello, las últimas cifras de cuota del mercado de MdD parecen señalar cierto estancamiento en los principales países europeos. Uno de los últimos estudios desarrollados por IRI a nivel europeo confirma que la MdD sufrió en 2014 un leve retroceso $(0,1 \%)$ en su nivel de ventas, lo que se tradujo, en algunos casos, en un estancamiento de las cifras de beneficio y la cuota de mercado de la marca propia. De acuerdo con dicho estudio, la principal razón para este descenso reside en las diferencias de precio cada vez menores que existen entre las marcas de distribuidor y las de fabricante ${ }^{5}$, aunque aspectos como el aumento de la actividad promocional por parte de las marcas nacionales y la dificultad para mantener el nivel de crecimiento que la MdD había alcanzado, pueden explicar, igualmente, la tendencia decreciente actual de las marcas propiedad del distribuidor.

5. Según Ignacio Larracoechea, presidente de Promarca, las marcas de fabricante han logrado mejorar su relación calidad-precio (El País, 2016). Esto, unido al incremento del precio medio de las MdD en un 2,2\% (año 2014 con respecto al año 2013), según señala un estudio de la Organización de Consumidores y Usuarios (OCU), explica por qué las marcas de fabricante han comenzado, lentamente, a recuperar parte del terreno perdido. 


\section{3. ¿Debe el minorista apostar por la MdD o debe mantener un número mínimo de marcas de fabricante?}

La lucha actual por rebajar los precios de venta ha provocado una importante reducción de los surtidos de muchos establecimientos minoristas (por ejemplo, Wal-Mart, Mercadona o Home Depot), que optaron a finales de 2008 por retirar referencias de sus lineales de venta (Steenkamp y Kumar, 2009), fundamentalmente aquellas con menor rotación y las que no respondían a necesidades reales de los consumidores. Al mismo tiempo, y siendo conscientes de que el surtido es una herramienta clave en el posicionamiento y la imagen de los minoristas, otros establecimientos (fundamentalmente supermercados e hipermercados), como por ejemplo Carrefour y Lidl, tomaron la decisión de ampliar sus surtidos y satisfacer todas las necesidades de los consumidores, no exclusivamente las básicas.

Los minoristas que ofrecen una combinación de marcas de fabricante y MdD esperan que el aumento de la variedad percibida en su surtido por parte del consumidor ayude a reducir su intención de cambiar de establecimiento. En cambio, en el caso de los minoristas que ofertan un surtido solo con MdD, se espera que no tenga influencia, ya que no hay variedad en un surtido que comprende una sola marca.

No obstante, la retirada de marcas de fabricante de los surtidos no siempre es sencilla. Hay numerosos ejemplos de situaciones en las que los minoristas, después de eliminar determinadas marcas nacionales, han tenido que rectificar esta decisión presionados por sus propios clientes. Por ejemplo, como apuntan Sloot y Verhoef (2008), la antigua cadena holandesa minorista de alimentación Edah decidió suprimir 2.000 artículos de marca de fabricante, para introducir 1.000 artículos de sus MdD. En respuesta a las muchas quejas de los clientes y al descenso de ventas en los seis meses posteriores a esta reducción del surtido, tuvieron que reintroducir en poco tiempo unos 1.000 productos, principalmente de marcas nacionales de alto valor. En esta misma línea, el minorista británico ASDA se negó a vender la marca Charmin (Procter \& Gamble), y los minoristas alemanes Edeka y Metro retiraron algunas marcas de fabricante de sus surtidos porque estaban insatisfechos con la política de precios y de distribución de los fabricantes (Sloot y Verhoef, 2011). Sin embargo, todos ellos se vieron obligados a reintroducir dichas marcas, aceptando las condiciones de los fabricantes, para poner fin al boicot de los consumidores y evitar un mayor daño a su imagen (Sloot y Verhoef, 2011).

Son diversos los motivos que la literatura aporta para defender que una retirada de marcas de fabricante de los surtidos de los minoristas puede dañar su imagen y sus ventas. Una de las causas esgrimidas es que un «surtido completo» debe incluir la mayoría de las marcas del mercado (Pepe et al., 2012), incluyendo las marcas renombradas (Sloot y Verhoef, 2008). Esto es especialmente relevante en determinados formatos comerciales, como hipermercados o supermercados; en el caso de los hard discount ${ }^{6}$, los consumidores asumen que su surtido tendrá una menor proporción de marcas de fabricante (Gázquez-Abad et al., 2015). Por otro lado, los minoristas deben tratar de rentabilizar sus lineales de venta incluyendo aquellas marcas de fabricante que se encuentran bien posicionadas entre los consumidores y con volumen medio-alto de ventas (Gómez y Rozano, 2009). La eliminación de una importante cantidad de marcas de fabricante para aumentar las ventas de las MdD puede ser percibida por los consumidores como un menor valor del surtido (Rubio et al., 2014), lo que puede afectar, como ya hemos comentado anteriormente, a la propia imagen del establecimiento. Para evitar esto, numerosos autores (entre ellos, González-Benito y Martos-Partal, 2010; Vilas, 2011) sugieren que los minoristas separen, en sus lineales de venta, las marcas nacionales de sus propias MdD. Los consumidores considerarán totalmente incompleto un surtido en el que todas las marcas de fabricante (especialmente las que son consideradas de alto valor) sean suprimidas, lo que puede provocar una decidida intención de cambio de establecimiento, al menos en el caso de los consumidores que muestran una alta preferencia por este tipo de marcas. En el caso de clientes habituales a las MdD, la eliminación de las marcas nacionales puede modificar su percepción sobre el surtido del minorista, si bien no modificará su elección de establecimiento, ni su comportamiento de compra.

6. El $80 \%$ de los productos que se pueden encontrar en los lineales de Lidl o Aldi son de marca propia (El Mundo, 2016). 


\section{Conclusiones}

La MdD es un elemento fundamental en la estrategia competitiva de los minoristas, especialmente en el contexto actual. La mejora del posicionamiento en la calidad de estas marcas, unido a los cambios en los patrones de consumo que se han producido en los últimos años de crisis económica, han animado a muchos minoristas a darle un mayor papel protagonista a la MdD en sus lineales, hasta el punto de que, en muchas ocasiones, han llegado a eliminar la totalidad de las marcas nacionales en algunas categorías de producto. Pero ¿es recomendable esta decisión? Siguiendo a Sloot y Verhoef (2011), es necesario que el minorista tenga en consideración algunos aspectos a la hora de plantearse la reducción de marcas en sus surtidos.

En primer lugar, es recomendable que de manera periódica el minorista revise sus surtidos a modo de mantenimiento y actualización, de manera que aquellas referencias que realmente no aporten valor sean eliminadas. No obstante, recomendamos al minorista que tenga cuidado y que no excluya del surtido marcas fuertes o hedónicas, puesto que los consumidores son muy sensibles a la eliminación de marcas de alto valor en categorías hedonistas, especialmente si se trata de establecimientos orientados a los servicios al cliente, y no tanto al precio. Además, los consumidores reaccionan con más fuerza a la eliminación total de una marca del surtido que a una reducción de diversas variedades y/o formatos de dicha marca. Como consecuencia, se recomienda a los minoristas que utilicen varios criterios económicos antes de decidir la supresión total de una marca; entre estos criterios deberá considerarse el valor de marca, la función del producto o el nivel de precios.

En resumen, siguiendo la recomendación de Gázquez-Abad et al. (2015), creemos que la mejor opción para el minorista es que cuente con surtidos en los que haya presencia tanto de marcas nacionales como de MdD, pues son los que van a generar una mayor lealtad al establecimiento y van a permitir llegar a consumidores de diferentes segmentos de mercado. Eso sí, para que los resultados obtenidos en esta «mezcla» sean óptimos, es necesario que el minorista sea capaz de construir una marca propia poderosa y que posea un posicionamiento en cuanto al valor lo más cercano posible a las marcas nacionales de mayor calidad.

\section{Bibliografía}

AlLAWADI, K. L.; KELLER, K. L. (2004). «Understanding retail branding: conceptual insights and research priorities». Journal of Retailing. Vol. 80, n. ${ }^{\circ} 4$, págs. 331-342.

AMROUCHE, N.; ZACCOUR, G. (2007). «Shelf-space allocation of national and private brands». European Journal of Operational Research. N. ${ }^{\circ}$ 180, págs. 648-663.

BALTAS, G.; ARGOUSLIDIS, C. (2007). "Consumer characteristics and demand for store brand». International Journal of Retail and Distribution Management. Vol. 3, n. ${ }^{\circ}$, págs. 328-341.

CASARES, J.; REBOLLO, A. (1996). Distribución Comercial. Madrid: Civitas.

DHAR, S. K.; HOCH, J. (1997). «Why Store Brand Penetration Varies by Retailer». Marketing Science. Vol. 16, n. ${ }^{\circ}$ 3, págs. 208-27.

EL MUNDO (2016). «Marcas blancas: la mejor opción». Disponible en línea [fecha de consulta: 9 de febrero de 2016]: <http://www.elmundo.es/vida-sana/estilo-y-gastro/2016/02/09/56b32e6246163f1f6a8b4611.html>.

EL PAÍS (2016). «¿Quién compra las marcas blancas? ¿Son cosa de ricos o de pobres?». Disponible en línea [fecha de consulta: 4 de marzo de 2016]: <http://economia.elpais.com/economia/2016/02/25/actualidad/ 1456397603_355036.html>.

GÁZQUEZ-ABAD, J. C.; MARTíNEZ-LÓPEZ, F. J.; MONDÉJAR-JIMÉNEZ, J. A.; ESTEBAN-MILLAT, I. (2015). "Mixed assortments vs. store brand-only assortments: The impact of assortment composition and consumer characteristics on store loyalty». Revista Española de Investigación en Marketing, ESIC. Vol.19, n. ${ }^{\circ}$, págs. 24-45.

GÁZQUEZ-ABAD, J. C.; SÁNCHEZ-PÉREZ, M. (2007). «Análisis de la estructura competitiva entre marcas nacionales y marcas privadas: un análisis empírico con datos de escáner». Información Comercial Española (ICE): Revista de economía. N. ${ }^{\circ} 839$, págs. 163-193. 
GÓMEZ, M.; ROZANO, M. (2009). «Marcas de distribuidor: análisis del espacio en el lineal por categorías y enseñas». Cuadernos de Gestión. Vol. 9, n. ${ }^{\circ}$ 1, págs. 81-98.

GÓNZALEZ-BENITO, O.; MARTOS-PARTAL, M. (2010). «Marcas de distribuidor y fidelización de clientes». En: Distribución comercial y comportamiento del consumidor. Oviedo: Cátedra Fundación Ramón Areces de Distribución Comercial, págs. 85-100.

GREWAL, D.; KRISHNAN, R.; BAKER, J.; BORIN, N. (1998). «The effect of store name, brand name and price discounts on consumers' evaluations and purchase intentions». Journal of Retailing. N. ${ }^{\circ} 74$ (3), págs. 331-352.

IRI (2013). Private label. Balancing quality and value. IRI Private Label Special Report. Disponible en línea [fecha de consulta: 4 de octubre de 2014]: <http://www.iriworldwide.eu/Portals/O/articlepdfs/PrivateLabel_2013_ Full\%20Report.pdf>.

IRI (2014). Private Label in Western Economies. IRI Private Label Special Report. Disponible en línea [fecha de consulta: 7 de marzo de 2016]: <http://www.iriworldwide.com/IRI/media/IRI-Clients/pl_report_final_7-Jan-15. pdf>.

IRI (2016). Infoscan census topline. 8 marzo. Disponible en línea [fecha de consulta: 8 de marzo de 2016]: <http:// www.iriworldwide.com/IRI/media/IRI-Clients/New_TopLine_1-2_Enero16_2.pdf>.

LAMEY, L.; DELEERSNYDER, B.; STEENKAMP, J-B.; DEKIMPE, M. (2012). «The effect of business-cycle fluctuations on private-label share: What has marketing conduct got to do with it?». Journal of Marketing. Vol. 76, enero, págs. 1-19.

PEPE, M. S.; ABRATT, R.; DION, P. (2012). «Competitive advantage, private-label brands and category profitability». Journal of Marketing Managemen. Vol. 28, n. ${ }^{\circ}$ 1-2, págs. 154-172.

PUELLES, J. A. (2011). «Introducción a las MDD: concepto, evolución, situación actual, tipología, marco legal e institutos de investigación». En: PUELLES, J. A.; GÓMEZ, M.; PUELLES, M. (coords.). Marcas de distribuidor. Concepto, evolución, protagonistas y adaptación a los ciclos económicos. Madrid: Pirámide, págs. 28-30.

PUELLES, J. A.; GÓMEZ, M.; PUELLES, M. (2011). Marcas de distribuidor. Concepto, evolución, protagonistas y adaptación a los ciclos económicos. Madrid: Pirámide.

PUELLES, J. A.; PUELLES, M. (2003). «Marcas de distribuidor: más de 30 años de un proceso dinámico, competitivo e imparable». Distribución y Consumo. Vol. 69, págs. 55-71.

ROGER, G. (2010). Las marcas distribuidor en el sector de la alimentación. Barcelona: EAE Business School, Strategic Research Center. Disponible en línea [fecha de consulta: 8 de marzo de 2016]: <http://recursos. anuncios.com/files/359/50.pdf>.

RUBIO, N.; VILLASEÑOR, N.; OUBIÑA, J. (2015). «Consumer identification with store brands: Differences between consumers according to their brand loyalty». Business Research Quarterly. Vol. 18, n. ${ }^{\circ}$ 2, págs.111-126.

SERRA, T.; PUELLES, J. A. (1993). «Análisis de las marcas de distribución en el mercado de la alimentación». Actas de los V encuentros de Profesores Universitarios de Marketing. Sevilla, págs. 103-108.

Sloot, L. M.; Verhoef, P. C. (2008). «THE IMPACT OF BRAND DELISTING ON BRAND SWITCHING and store switching intentions». Journal of Retailing. Vol. 84, n. ${ }^{\circ}$ 3, págs. 281-296.

SLOOT, L. M.; Verhoef, P. C. (2011). «Reducing assortments without losing business. Key lessons for retailers and manufacturers». New Strategies. Vol. 3, n. ${ }^{\circ}$ 2, págs. 27-33.

STEENKAMP, E. M.; Kumar, N. (2009). «Don't be undersold». Harvard Business Review. Vol. 87, n. 12, págs. 90-95.

VILAS, J. M. (2011). «Marcas líderes y distribuidores. Buenas prácticas de colaboración». Distribución y Consumo. N. ${ }^{\circ} 117$, págs. 23-31. 


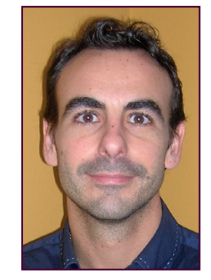

Juan Garlos Gázquez Abad

jgazqueza@uoc.edu

Profesor titular de la Universidad de Almería.

Profesor colaborador de los Estudios de Economía y Empresa (UOC)

Consultor de los Estudios de Economía y Empresa de la Universitat Oberta de Catalunya, profesor titular de universidad del área de Comercialización e Investigación de Mercados de la Universidad de Almería. Es doctor por la Universidad de Almería. Ha sido profesor visitante en la Universidad de Gante (Bélgica). Los resultados de sus investigaciones han sido publicados en múltiples revistas internacionales y nacionales de impacto. Igualmente, es autor de diversos libros y capítulos de libros internacionales y nacionales relacionados con la gestión minorista y la marca de distribuidor. Es co-chair de la Research on National Brand \& Private Label Marketing International Conference, congreso internacional sobre marcas nacionales y marcas de distribuidor que se celebra anualmente en Barcelona y que está esponsorizado por la UOC.

Los textos publicados en esta revista están -si no se indica lo contrario- bajo una licencia Reconocimiento-Sin obras derivadas 3.0 España de Creative Commons. Puede copiarlos, distribuirlos y comunicarlos públicamente siempre que cite su autor y la revista y la institución que los publica (autoría, nombre de la revista, institución editora); no haga con ellos obras derivadas. La licencia completa se puede consultar en http://creativecommons.org/licenses/by-nd/3.0/es/deed.es.

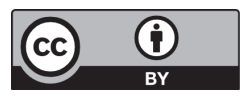

